\title{
Unidirectional waves over slowly varying bottom Part II. Quasi-homogeneous approximation of distorting waves *
}

\author{
S.R. Pudjaprasetya ${ }^{a}$, E. van Groesen ${ }^{b, *}$ \\ a Institut Teknologi Bandung, Department of Mathematics, Indonesia \\ b University of Twente, Department of Applied Mathematics. The Netherlands
}

Received 16 December 1994; revised 29 June 1995

\begin{abstract}
A new Korteweg-de Vries type of equation for uni-directional waves over slowly varying bottom has been derived in Part I. The equation retains the Hamiltonian structure of the underlying complete set of equations for surface waves. For flat bottom it reduces to the standard Korteweg-de Vries equation. Uniform travelling waves (solitary and cnoidal waves) that exist when the bottom is flat will distort over a varying bottom. In this paper, the distortion of periodic and solitary travelling waves will be studied. The distortion is in the first instant approximated by a quasi-homogeneous succession of uniform waves, each one being determined by specifying the horizontal momentum (and hence the amplitude) at the location of the wave. The changing value of the momentum with position is found first from energy conservation. For periodic, cnoidal waves, for which the mass vanishes, the change of wavelength has to be taken into account; some numerical results are given. Solitary waves carry a mass that depends on the amplitude (momentum) and the quasi-homogeneous approximation has to be modified to satisfy mass-conservation. This is achieved by introducing an additional parameter in the base functions with which the distortion is approximated. Instead of using pure solitary waves, one modification consists of adding a tail of finite, but varying length and amplitude. When the bottom decreases sufficiently fast far away from the wave, an alternative description of the distortion will be presented as a succession of solitary waves above a varying, non-flat equilibrium elevation of the surface. In both cases, the dynamic equations obtained from energy and mass conservation differ in essential order from the result without modification.
\end{abstract}

\section{Introduction}

We start with a short description of (the derivation of) the equation that was obtained in Part I [5], and that will be studied in this paper. Consider a layer of ideal fluid (incompressible and irrotational) above topography $h(x)$, see Fig. 1. Let $\eta(x, t)$ be the wave height measured from an undisturbed water level and

\footnotetext{
* Part of the research is sponsored by the commission of the European Communities, Directorate General XII-B, Joint Research project CI1*-CT93-0018, between the Department of Mathematics, Institut Teknologi Bandung, and the Faculty of Applied Mathematics, University of Twente.

* Corresponding author.
} 


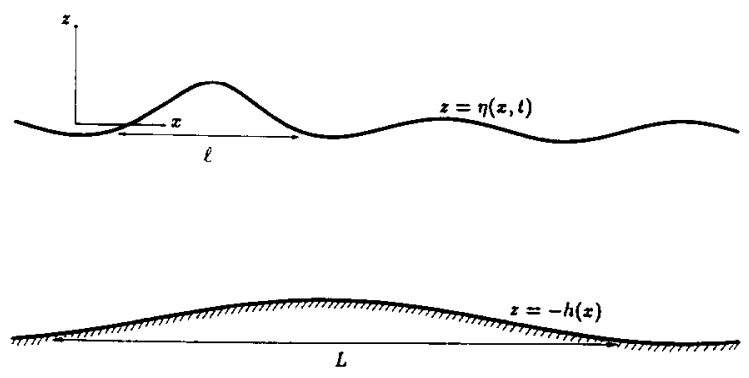

Fig. 1. Fluid domain.

$u(x, t) \equiv \Phi_{x}(x, z=\eta(x, t), t)$, where $\Phi(x, z, t)$ is the velocity potential. The full set of equations for the gravity driven surface waves can be described as a Hamiltonian system (cf. Refs. [10,1,2]) in the following way

$$
\partial_{t}\left(\begin{array}{l}
u \\
\eta
\end{array}\right)=\left(\begin{array}{cc}
0 & -\partial_{x} \\
-\partial_{x} & 0
\end{array}\right)\left(\begin{array}{c}
\delta_{u} \breve{H} \\
\delta_{\eta} \breve{H}
\end{array}\right),
$$

with total energy of the system $\breve{H}(u, \eta)$ as the Hamiltonian. Considering rather long and rather low waves, and for slow bottom variations

$$
h^{\prime}(x)=\mathcal{O}(\epsilon),
$$

an approximation of the total energy was obtained. Substituting the approximate energy into Eq. (1) gives Boussinesq-type of equations. These are bi-directional wave equations. By restricting to uni-directional waves, taking into account effects of bottom reflections, (forced) first order in time scalar equations are obtained. In particular, for waves above a flat bottom, $h(x)=h_{0}$, uni-directional waves were found from Eq. (1) that are described by the familiar Korteweg-de Vries (KdV) equation. This equation is written as a Hamiltonian system like

$$
\partial_{r} \eta=-c_{0} \partial_{x} \delta_{\eta} H_{0}(\eta)
$$

correct up to order $\mathcal{O}(\epsilon)$. Here, $c_{0}=\sqrt{g h_{0}}$, and $g$ is the gravitational acceleration. The Hamiltonian $H_{0}$ is ( $1 / 2 \mathrm{~g}$ times) the total energy of the system:

$$
H_{0}(\eta)=\int \frac{1}{2} \eta^{2}+\epsilon\left(-\frac{1}{12} h_{0}^{2} \eta_{x}^{2}+\frac{\eta^{3}}{4 h_{0}}\right)
$$

In this paper, we concentrate on the case when the bottom variations are very mild. In that case effects of reflected waves can be neglected and the governing equation can again be written as a Hamiltonian system like

$$
\partial_{t} \eta=-\Gamma(x) \delta_{\eta} H(\eta),
$$

correct up to $\mathcal{O}(\epsilon)$. Here appears a skew-symmetric operator given by

$$
\Gamma(x)=\frac{1}{2}\left(c(x) \partial_{x}+\partial_{x} c(x)\right) \quad \text { with } \quad c(x)=\sqrt{g h(x)},
$$

and the Hamiltonian

$$
H(\eta)=\int \frac{1}{2} \eta^{2}+\epsilon\left(-\frac{1}{12} h^{2}(x) \eta_{x}^{2}+\frac{\eta^{3}}{4 h(x)}\right),
$$


which is ( $1 / 2 g$ times) the uni-directionalised energy. We call Eq. (4) above the $K d V$-top equation: it reduces to the familiar $\mathrm{KdV}$ if the bottom is flat, $h(x)=h_{0}$, but else incorporates the effects of the topography.

The standard KdV equation (2) admits uniform travelling waves (solitary waves and cnoidal waves) that propagate at constant speed undisturbed in shape. The existence of such solutions is a consequence of translation symmetry. The shape and the propagation speed are uniquely determined by the (constant) value of the momentum. In fact, the wave shape maximizes the energy at the given value of the momentum, a property that will be used in the following.

For varying bottom there is no translation symmetry, and cnoidal and solitary waves will distort. The aim of this paper is to derive approximate descriptions for the distortion of such waves. To that end we start with the simple idea of a quasi-homogeneous approximation with undisturbed waves. This means that at a certain time, when the distorting wave is positioned at a certain place $\varphi$, its shape will be approximated by that of a pure travelling wave of the KdV equation that applies when the bottom is flat and at a depth $h(\varphi)$ that is determined by the position. To describe the succession, let $S(\varphi, \gamma)(x-\varphi)$ denote the travelling wave centered at $x=\varphi$ (above a flat bottom at depth $h(\varphi)$ ) that has momentum $\gamma$. Then a 2-parameter family of travelling waves is obtained:

$$
\mathcal{N}=\{S(\varphi, \gamma)(x-\varphi) \mid \gamma, \varphi \in R\}
$$

Starting with a pure travelling wave, i.e. with an initial condition on the manifold $\mathcal{N}$, an approximate solution of Eq. (4) will first be sought as an evolution on that manifold, i.e. as a quasi-homogeneous succession of uniform travelling waves:

$$
t \longmapsto S(\varphi(t), \gamma(t))(x-\varphi(t)) .
$$

Here, $\varphi(t)$ and $\gamma(t)$ have to be determined as functions of time. Specifying them implies that the successive wave profiles and the varying propagation speed are determined. The dynamic equations for $\gamma(t)$ and $\varphi(t)$ are obtained by requiring that the energy is conserved during the succession. This is motivated by the fact that for all solutions of KdV-top this property holds. Doing so, it will follow that the amplitude is increasing and the velocity is decreasing for solitary waves during run up.

The approximation described above is in several aspects too simple and has to be modified somewhat. First, for periodic waves, the wavelength changes with bottom variations. The change in wavelength can be determined a priori from an investigation of the lowest order (linear) approximate equation. This effect can be dealt with by a simple scaling of the spatial variable, the scaling being determined by the bottom variations. Secondly, for solitary waves, a modification is needed to guarantee that the total mass is conserved in the required order. (In the periodic case, waves that are used in the approximation all have vanishing mass, and hence mass conservation is automatically satisfied.) To explain this, it must be observed that the KdV-top equation (4), has a mass-like quantity that is conserved during the evolution. This mass functional is given by

$$
\int \frac{\eta}{\sqrt{c(x)}}
$$

In fact, it is a so-called Casimir functional that exists (irrespective of the property of the Hamiltonian) because the kernel of the skew-symmetric operator $\Gamma(x)$ in Eq. (5) is not trivial. This generalizes the corresponding property for the $\mathrm{KdV}$ equation above a flat bottom. The consequence is that the functions

$$
\eta_{\beta}(x)=\frac{\beta}{\sqrt{c(x)}}, \quad \text { for } \beta=\mathcal{O}(\epsilon)
$$

are equilibrium solution of the KdV-top equation, since their contribution in the Boussinesq-terms are in a negligible order of the equation. This is certainly different from the usual KdV-equation, for which only the flat surface $\eta \equiv 0$ is an equilibrium. The generalized equilibrium surfaces $\eta_{\beta}$ may be physically relevant when the bottom topography is such that they decay to zero sufficiently fast, i.e. if $c(x) \longrightarrow \infty$ for $|x| \longrightarrow \infty$. 
In a quasi-homogeneous approximation with cnoidal waves, this mass functional is zero all the time, as stated above. However, for a quasi-homogeneous approximation with solitary waves as described above, the mass functional is decreasing for waves during run up. This is caused by the fact that while growing, the mass of the solitary waves decreases. Hence, the succession as proposed cannot take all the amount of water which is accumulated by the changing depth.

So, we need a modification of the method proposed above. The modification should be based on the two assumptions i.e. energy and mass conservation. For a quasi-homogeneous approximation without modification we only have one parameter (the momentum) as a function of the position, and so, clearly it is not possible to satisfy energy and mass conservation. As a consequence, the set of base functions has to be enlarged and to contain an additional parameter. The set of functions to be taken is not prescribed in advance, however, it should be determined from mathematical-physical arguments. In this paper we show that two reasonable choices can be made. The first one is to introduce a tail behind the solitary wave. The appearance of the tail for an initial solitary wave under a perturbed $\mathrm{KdV}$ equation is observed in literature; results in [7] and [8] that are based on the inverse scattering method, for instance, show that the tail directly behind the solitary wave has a shape of a plateau. Motivated by these results, we choose a compact support tail of uniform height. The height of the tail $\rho$, then serves as a parameter that can be adjusted to comply with mass conservation. Similar - but different - way of attacking the problem concerning mass conservation was done by Newell [9]. A second method, somewhat strange but at least reasonable from a mathematical point of view, is to consider the solitary wave not above a flat surface, but above the generalized equilibrium surfaces described above. By varying the amplitude factor $\beta$ of the equilibrium surfaces, mass changes can be incorporated (provided the equilibrium surfaces contain a finite total mass, which requires some conditions on the topography). Even though the tail and the equilibrium surface has amplitude of high order $\mathcal{O}(\epsilon)$, over long time it makes an order one contribution to the mass. In both cases it is essential to deal with the two parameters at the same time to invoke energy and mass conservation. For the method that uses the tail-approximation, the result differs in that respect from the one that is found by Newell, for instance.

The organization of the paper is as follows. In Section 2 we derive, starting from the original problem, two physical quantities, the momentum and mass functionals. For uni-directional waves above even bottom, both are conserved quantities. For uneven bottom, the momentum functional is no longer conserved. In Section 3 the distortion of solitary waves will be investigated using the simple attack described above. The problem concerning mass conservation for the quasi-homogeneous approximation with solitary waves will be tackled in Section 4. Section 5 deals with periodic waves; the wavelength adaption is described and results from numerical computations are shown. In an appendix, the technical proof of a theorem about the existence of solitary wave solutions of Eq. (4) above a generalized equilibrium surface is given.

\section{Momentum and mass functionals}

For the complete problem in $\eta, u$-variables (before uni-directionalisation), the horizontal momentum is given hy

$$
I_{\mathrm{hor}}=\iint_{-h(x)}^{\eta(x, t)} \Phi_{x} \mathrm{~d} z \mathrm{~d} x=\int \eta u \mathrm{~d} x+\int-h^{\prime}(x) \Phi(x,-h(x)) \mathrm{d} x
$$

The time derivative of the momentum-like functional defined by $l(u, \eta) \equiv \int u \eta$, for solutions of $(1)$ is

$$
\partial_{t} \breve{I}(u, \eta)=\left\langle\delta_{u} \check{I}, u_{t}\right\rangle+\left\langle\delta_{\eta} \breve{I}, \eta_{t}\right\rangle=\left\langle\eta,-\partial_{x} \delta_{\eta} \breve{H}\right\rangle+\left\langle u,-\partial_{x} \delta_{u} \breve{H}\right\rangle=\left\langle\delta_{\eta} \breve{H}, \partial_{x} \eta\right\rangle+\left\langle\delta_{u} \breve{H}, \partial_{x} u\right\rangle
$$


This expression vanishes when the Hamiltonian $\breve{H}(u, \eta)$ is translation invariant, which is the case if the density does not depend explicitly on $x$. When the bottom is flat, $\not h(u, \eta)$ is translation invariant and the momentum functional $\breve{I}(u, \eta)$ is conserved.

In Ref. [5] a transformation is introduced from $u, \eta$ variables to variables that account in lowest order for right and left running waves, respectively. Let $\bar{I}(\eta)$ denote the momentum functional that is obtained from $\breve{I}(u, \eta)$ when restricted to uni-directional waves, explicitly

$$
\bar{l}(\eta)=\int \sqrt{\frac{g}{h(x)}} \eta^{2} .
$$

Therefore, omitting a factor $1 / 2 \mathrm{~g}$, we take as momentum functionals for the case of even and uneven bottom

$$
I_{0}(\eta)=\int \frac{1}{2} \frac{\eta^{2}}{c_{0}} \mathrm{~d} x \text { and } I(\eta)=\int \frac{1}{2} \frac{\eta^{2}}{c(x)} \mathrm{d} x,
$$

respectively. Clearly, $I_{0}(\eta)$ is conserved for solutions of Eq. (2), but $I(\eta)$ is not conserved for solutions of Eq. (4) since

$$
\partial_{t} I(\eta)=\left\langle\frac{\eta}{c(x)},-\Gamma(x) \delta_{\eta} H\right\rangle=\left\langle\Gamma(x)\left(\frac{\eta}{c(x)}\right), \delta_{\eta} H\right\rangle,
$$

which does not vanish in general.

A (generalised) Hamiltonian system may have Casimir functionals related to the fact that the structure map of the system is degenerate. For the complete system (1), and for the Boussinesq approximations there are two Casimir functionals: $\int u$ and $\int \eta$. The reason is that their variational derivative are constant, and vanish after application of $\partial_{x}$. The KdV-top equation (4) has as Casimir functional

$$
C(\eta)=\int \frac{\eta}{\sqrt{c(x)}}
$$

since $\Gamma(x)$ in $\mathrm{Eq} .(5)$ annihilates its variational derivative:

$$
\Gamma(x) \frac{1}{\sqrt{c(x)}}=0 .
$$

The Casimir $C(\eta)$ generalises the mass-like Casimir functional for the $\mathrm{KdV}$ equation

$$
C_{0}(\eta)=\int \frac{\eta}{\sqrt{c_{0}}}
$$

\section{Quasi-homogeneous approximation with solitary waves}

The idea of the quasi-homogeneous approximation is the following. When at a certain time the wave is at $x=\varphi$, the wave shape at that place is approximated by a uniform solitary wave, with a certain momentum $\gamma$, above flat bottom at depth $h(\varphi)$, see Fig. 2. So, we need to have expressions for the uniform solitary waves. The $\mathrm{KdV}$ equation for flat bottom $h(x)=h(\varphi)$ is

$$
\partial_{t} \eta=-c(\varphi) \partial_{x} \delta_{\eta} \bar{H}(\varphi, \eta), \quad c(\varphi)=\sqrt{g h(\varphi)},
$$

with Hamiltonian

$$
\bar{H}(\varphi, \eta)=\int \frac{1}{2} \eta^{2}+\epsilon\left(-\frac{1}{12} h(\varphi)^{2} \eta_{x}^{2}+\frac{\eta^{3}}{4 h(\varphi)}\right)
$$




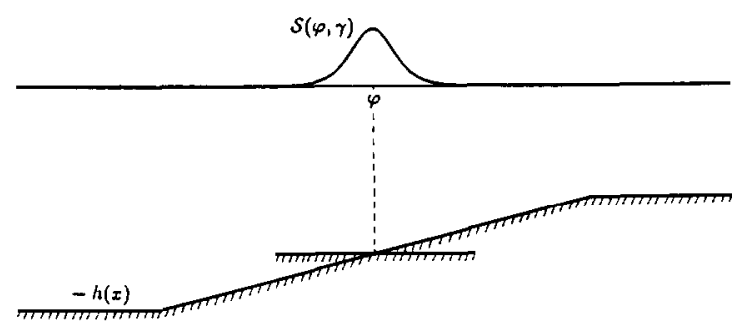

Fig. 2. The uniform solitary wave $\mathcal{S}(\varphi, \gamma)$ above flat bottom $h(x)=h(\varphi)$ approximates the wave shape at $x=\varphi$.

For the KdV equation (14), the homogeneous momentum functional

$$
\vec{I}(\varphi, \eta)=\int \frac{\eta^{2}}{2 c(\varphi)}
$$

is conserved. It is well known that Eq. (14) has solitary wave solutions. These can in fact be characterised as solutions of the constrained maximisation problem of maximising the energy at the prescribed value of momentum. More precisely, with $X_{\infty}=\{\eta: R \rightarrow R \mid \eta$ and its derivative tend to zero as $x \rightarrow \pm \infty\}$ as function space, and a given value $\gamma$ of the momentum, we consider

$$
\max _{\eta}\left\{\bar{H}(\varphi, \eta) \mid \bar{I}(\varphi, \eta)=\gamma, \quad \eta \in X_{\infty}\right\}
$$

Solutions of this constrained maximisation problem satisfy the Lagrange multiplier rule, i.e. the equation

$$
\delta_{\eta} \bar{H}=\lambda \delta_{\eta} \bar{I}, \quad \text { for some } \lambda \in R,
$$

explicitly

$$
\eta+\epsilon\left(\frac{1}{6} h(\varphi)^{2} \eta_{x x}+\frac{3}{4 h(\varphi)} \eta^{2}\right)=\frac{\lambda}{c(\varphi)} \eta
$$

This equation is indeed easily recognised as the equation for solitary wave solutions of Eq. (14) with velocity $\lambda$. Moreover, since the $I$-flow is translation in the $x$-space, any translation of the solution of Eq. (17) is also a solution, representing wave profiles at different positions. We will denote the solitary wave that is centred at $x=\varphi$, by $\mathcal{S}(\varphi, \gamma)(x-\varphi)$. This function can be written down explicitly; in the desired variables it reads

$$
\mathcal{S}(\varphi, \gamma)(x-\varphi)=a \operatorname{sech}^{2} b(x-\varphi)
$$

with

$$
a(\varphi, \gamma)=3 g\left(\frac{\gamma}{4}\right)^{2 / 3} c(\varphi)^{-4 / 3}, \quad b(\varphi, \gamma)=\frac{3}{2} g^{2}\left(\frac{\gamma}{4}\right)^{1 / 3} c(\varphi)^{-11 / 3}
$$

The velocity of this solitary wave is

$$
\lambda(\varphi)=c(\varphi)+\frac{3}{2} \epsilon g^{2}\left(\frac{\gamma}{4}\right)^{2 / 3} c(\varphi)^{-7 / 3}
$$

There is a simple relation between the velocity $\lambda$ and the so-called value function, which is the constrained maximum value of the optimisation problem, i.e. $\mathcal{H}(\varphi, \gamma)=\bar{H}(\varphi, \mathcal{S}(\varphi, \gamma))$. The velocity is the derivative with respect to $\gamma$ of this value function, as can be seen from

$$
\frac{\partial \mathcal{H}}{\partial \gamma}=\left\langle\delta_{\eta} \bar{H}, \mathcal{S}_{\gamma}\right\rangle=\lambda\left\langle\delta_{\eta} \bar{I}, \mathcal{S}_{\gamma}\right\rangle=\lambda \frac{\mathrm{d} \bar{I}}{\mathrm{~d} \gamma}=\lambda
$$




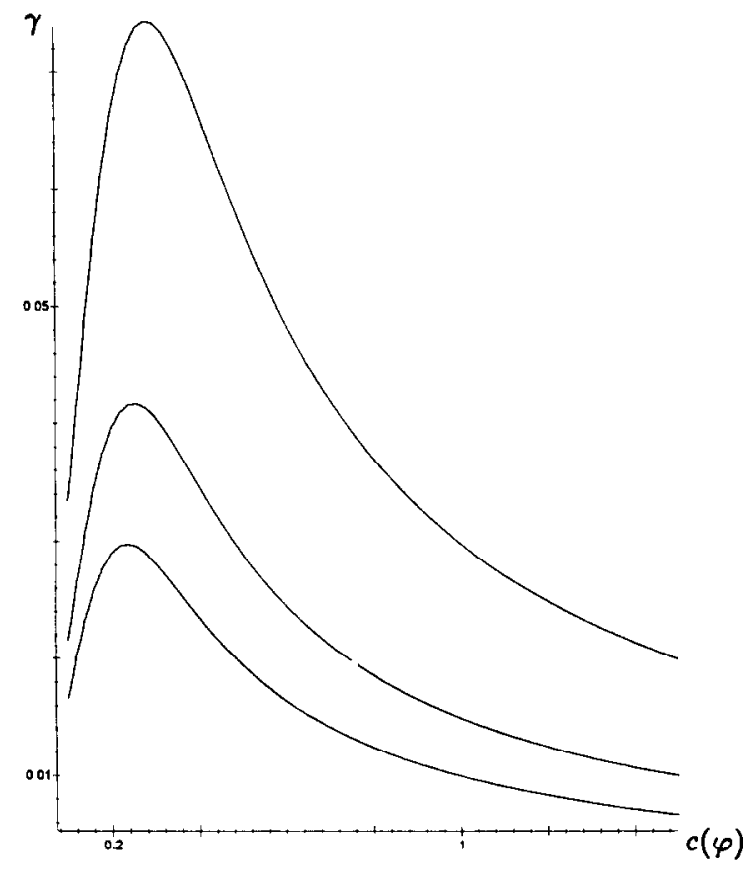

Fig. 3. Level sets of energy for $\gamma$ vs. $c=c(\varphi)$.

Using this result, the value function $\mathcal{H}(\varphi, \gamma)$ can be found by integrating Eq. (21) with respect to $\gamma$ :

$$
\mathcal{H}(\varphi, \gamma)=c(\varphi) \gamma+\frac{18}{5} \epsilon g^{2}\left(\frac{\gamma}{4}\right)^{5 / 3} c(\varphi)^{-7 / 3}
$$

Several lcvel sets of $\mathcal{H}$ are shown in Fig. 3. From this figure some characteristic properties for the quasihomogeneous approximation can be derived. Indeed, for such an approximation, the energy is taken to be constant. Its initial value then determines the specific level set, and the value of the momentum parameter can be read off as a function of the position $\varphi$ at $c(\varphi)$. Note that the level lines indicate that for large values of $c(\varphi)$, the momentum $\gamma$ increases with decreasing $c(\varphi)$, while for small values of $c(\varphi), \gamma$ decreases with decreasing $c(\varphi)$.

For the quasi-homogeneous approximation, energy conservation immediately leads to a differential equation for $\gamma$ as a function of position: differentiating $\mathcal{H}(\varphi, \gamma)=$ constant with respect to $\varphi$, there results

$$
\frac{\mathrm{d}}{\mathrm{d} \varphi} \mathcal{H}(\varphi, \gamma)=\mathcal{H}_{\varphi}+\mathcal{H}_{\gamma} \gamma_{\varphi}=0
$$

Substituting Eq. (22) gives the first order dynamics

$$
\gamma_{\varphi}=-\frac{c^{\prime}(\varphi) \gamma}{c(\varphi)}+\mathcal{O}(\epsilon)
$$

We can also calculate the evolution of $\gamma$ directly from the evolution of the momentum for exact solutions of KdV-top. The next calculation is correct up to somewhat higher order, $\mathcal{O}\left(\epsilon^{3}\right)$, for a reason that will become clear later. For an arbitrary solution, we obtain

$$
\frac{\mathrm{d}}{\mathrm{d} t} I(\eta)=\left\langle\frac{\eta}{c(x)}, \eta_{t}\right\rangle=\left\langle\frac{\eta}{c(x)},-\Gamma(x) \delta_{\eta} H\right\rangle=-\left\langle\eta,\left(\partial_{x}+\frac{1}{2} \frac{c^{\prime}(x)}{c(x)}\right) \delta_{\eta} H\right\rangle
$$




$$
\begin{aligned}
= & -\left\langle\eta, \partial_{x} \delta_{\eta} H\right\rangle-\left\langle\frac{1}{2} \frac{c^{\prime}(x)}{c(x)} \eta, \delta_{\eta} H\right\rangle \\
= & -\left\langle\eta, \partial_{x} \delta_{\eta} \bar{H}(\varphi, \eta)\right\rangle-\frac{\epsilon}{6}\left\langle\eta, 4 h h^{\prime} \eta_{x x}+2 h h^{\prime}(x-\varphi) \eta_{x x x}\right\rangle \\
& -\frac{3}{4} \epsilon\left\langle\eta,-2 h^{\prime} h^{-2}(x-\varphi) \eta \eta_{x}-h^{\prime} h^{-2} \eta^{2}\right\rangle-\frac{1}{2} \frac{c^{\prime}}{c}\left\langle\eta, \delta_{\eta} \bar{H}(\varphi, \eta)\right\rangle \\
& -\frac{1}{2}\left(c^{\prime \prime} c^{-1}-\left(c^{\prime}\right)^{2} c^{-2}\right)\langle(x-\varphi) \eta, \eta\rangle+\mathcal{O}\left(\epsilon^{3}\right) \\
\stackrel{(*)}{=} & -c^{\prime} \gamma-\frac{17}{12} \epsilon \frac{1}{g^{2}} c^{3} c^{\prime}\left\langle\eta, \eta_{x x}\right\rangle+\frac{9}{8} \epsilon g c^{-3} c^{\prime}\left\langle\eta, \eta^{2}\right\rangle-\frac{2}{3} \epsilon \frac{1}{g^{2}} c^{3} c^{\prime}\left\langle\eta,(x-\varphi) \eta_{x x x}\right\rangle \\
& +3 \epsilon g c^{-3} c^{\prime}\left\langle\eta^{2},(x-\varphi) \eta_{x}\right\rangle+\mathcal{O}\left(\epsilon^{3}\right) .
\end{aligned}
$$

Here, and also in the rest of the paper, $h, h^{\prime}, c, c^{\prime}, c^{\prime \prime}$ stand for $h(\varphi), h^{\prime}(\varphi), c(\varphi), c^{\prime}(\varphi), c^{\prime \prime}(\varphi)$, unless otherwise stated. To explain step $(*)$, observe that the first term is zero because of translation symmetry and the fifth term is zero because it is an integration of an odd function around $x=\varphi$ over the whole real line. Hence, the dynamic equation for $\gamma$ can be obtained from $\dot{\gamma}=\left.(\mathrm{d} / \mathrm{d} t) I(\eta)\right|_{\eta=\mathcal{S}}$, with $\mathcal{S}(\varphi, \gamma)$ given in Eq. (19). Calculations yield

$$
\dot{\gamma}=-c^{\prime} \gamma+\frac{42}{5} \epsilon g^{2}\left(\frac{\gamma}{4}\right)^{5 / 3} c^{-10 / 3} c^{\prime}+\mathcal{O}\left(\epsilon^{3}\right)
$$

The right hand side of Eq. (24) is actually $-\mathcal{H}_{\varphi}$, correct up to a negligible order, in this case $\mathcal{O}\left(\epsilon^{3}\right)$. The dynamics in $t$ can be obtained by differentiating the value function with respect to $t$ :

$$
\mathcal{H}_{\varphi} \dot{\varphi}+\mathcal{H}_{\gamma} \dot{\gamma}=0
$$

Solutions of Eq. (25) are

$$
\left(\begin{array}{l}
\dot{\gamma} \\
\dot{\varphi}
\end{array}\right)=\left(\begin{array}{rr}
0 & -1 \\
1 & 0
\end{array}\right)\left(\begin{array}{l}
\mathcal{H}_{\gamma} \\
\mathcal{H}_{\varphi}
\end{array}\right)
$$

except for some factor of proportionality $p(t)$ which is actually one in the prevailing order, since we have Eq. (24). The interesting thing to observe is that Eq. (26) is a set of classical Hamiltonian equations for $\gamma$ and $\varphi$, with $\mathcal{H}$ as the Hamiltonian.

Note that $\dot{\varphi}$, the velocity of the distorted solitary wave, is approximately $\lambda(\varphi)$; explicitly it is given by

$$
\dot{\varphi}=\lambda(\varphi)+\mathcal{O}\left(\epsilon^{2}\right)=c+\frac{3}{2} \epsilon g^{2}\left(\frac{\gamma}{4}\right)^{2 / 3} c^{-7 / 3}+\mathcal{O}\left(\epsilon^{2}\right)
$$

Note that in order to obtained relation Eq. (27), the calculation for $\dot{\gamma}$ in Eq. (24) had to be correct up to $\mathcal{O}\left(\epsilon^{3}\right)$. It is clearly seen from Eqs. (24) and (27) that the momentum (and hence the amplitude) of a solitary wave during run up increases and the velocity decreases. This agrees with common expectations. One observes also that when the bottom is flat $\left(c^{\prime}=0\right), \gamma$ is constant from translation symmetry - also from Eq. (24) and $\dot{\varphi}$ is exactly the velocity of the solitary wave $\lambda(\varphi)$.

Remark: In the quasi-homogeneous method described above, the parameters $\varphi$ and $\gamma$ that determine the wave, only depend on the depth at the position of the wave $h(\varphi)$ and not on the steepness of the bottom. But the transition time, i.e. the time that is needed for a soliton to travel (run up hill) along a certain distance, will in 
general depend on the bottom profile. This can be seen as follows. Let $\mathcal{T}$ denote the transition time from two arbitrary positions, $\varphi_{1}$ till $\varphi_{2}$. The relation between transition time $\mathcal{T}$ and the topography can be obtained from Eq. (27). Taking only the order one term in Eq. (27), we have

$$
\begin{aligned}
& \mathrm{d} t=\frac{1}{\dot{\varphi}} \mathrm{d} \varphi+\mathcal{O}(\epsilon), \\
& \mathcal{T}=\int_{\varphi_{1}}^{\varphi_{2}} \frac{1}{c(\varphi)} \mathrm{d} \varphi+\mathcal{O}(\epsilon) .
\end{aligned}
$$

From this explicit expression it is clear that the transition time depends on the bottom profile.

\section{Modified description of the distortion of solitary waves}

First let us see how the Casimir functional $C(\eta)$ changes when $\eta$ is approximated by the quasi-homogeneous succession found in the previous section. Its value at $\mathcal{S}(\varphi, \gamma)$ is found to be

$$
C(\mathcal{S}(\varphi, \gamma))=\frac{4}{g}\left(\frac{\gamma}{4}\right)^{1 / 3} c(\varphi)^{11 / 6}+\mathcal{O}\left(\epsilon^{2}\right)
$$

Differentiating with respect to $\varphi$ and substituting Eq. (23) will give

$$
\frac{\partial}{\partial \varphi} C(\mathcal{S}(\varphi, \gamma))=\frac{22}{3 g}\left(\frac{\gamma}{4}\right)^{1 / 3} c(\varphi)^{5 / 6} c^{\prime}(\varphi)+\mathcal{O}\left(\epsilon^{2}\right)
$$

This shows that the Casimir is not conserved: for a wave running up it decreases in the essential order $\mathcal{O}(\epsilon)$. The momentum (not a conserved quantity) is also changing in that order. In the following subsections two modifications of the approximation will be described that will enable us to regain mass conservation. The basic idea is to put the extra mass in a kind of shelf that is described with one parameter, its amplitude. This amplitude is of order $\mathcal{O}(\epsilon)$, but over long time it makes an order one contribution to the mass.

\subsection{Solitary waves with finite tail}

One way to modify the wave forms that are used in the quasi-homogeneous approximation is to introduce a tail of finite length behind the solitary wave profiles used above. For mild bottom variations there is analytical and numerical evidence, see [7] and [8], that the appearance of a tail is observed. The tail will be of finite length when the wave starts above a flat bottom and runs towards bottom variations. To be more precise, imagine a solitary wave above a flat bottom that is running to the right and starts to meet bottom variations, for instance a shoal, at $x=0$. Then, the mass-like Casimir functional starts to change, to decrease. To be able to compensate for the remaining mass, introduce a tail at the back of the wave. A similar idea has already been introduced by Newell, in [9], but here we construct the tail in a different way. The tail is chosen a priori of constant height, and (from causality) to be non zero only for a finite interval within $x<\varphi$. The position of the left end point of the tail will be determined by the lowest order theory: the position as predicted by the linearised (uneven bottom) equation for long waves. The linearised equation above topography has been studied in depth in Part I, and it reads

$$
\eta_{t}=-\Gamma(x) \eta
$$

The general solution can be written down explicitly: for any function $g$,

$$
\eta(x, t)=\frac{1}{\sqrt{c(x)}} g(\Theta)
$$




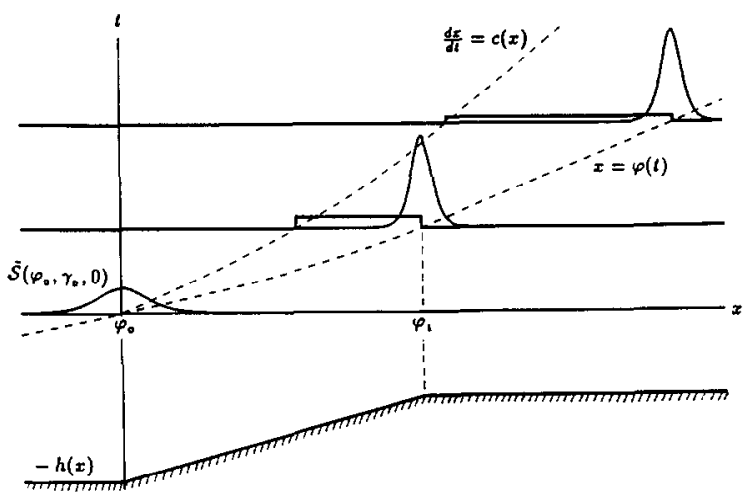

Fig. 4. Distorted solitary wave with finite tail.

with

$$
\Theta=\sigma(x)-t, \quad \sigma(x)=\int^{x} \frac{1}{c(\mu)} \mathrm{d} \mu .
$$

Stated differently, for any solution it holds that $\sqrt{c(x)} \eta(x, t)=g(\Theta)$ is constant along the right going characteristic $\Theta$. Hence, for the construction suggested above, the characteristic $\Theta=0$ (or $x=\sigma^{-1}(t)$ or $\mathrm{d} x / \mathrm{d} t=c(x)$ ) is the path for the left end point of the tail. So, the tail spans from $\Theta=0$ (or $x=\sigma^{-1}(t)$ ), till $x=\varphi(t)$, the present location of the solitary wave, see Fig. 4. The solitary wave with a tail of uniform height $\rho$ is then taken as a simple superposition:

$$
\tilde{S}(\varphi, \gamma, \rho)=\mathcal{S}(\varphi, \gamma)+\rho_{\chi}(\varphi ; x, t) \quad \text { with } \quad \chi(\varphi ; x, t)= \begin{cases}1, & \sigma^{-1}(t)<x<\varphi(t) \\ 0, & \text { elsewhere }\end{cases}
$$

The height of the tail is not determined in advance but will be taken to satisfy the energy and mass conservation. From Eq. (30) it follows that the mass that should be taken by the tail is of order $\mathcal{O}(\epsilon)$, so $\rho$ will be of order $\mathcal{O}(\epsilon)$ too.

In the following, we use the functions $\mathcal{S}(\varphi, \gamma, \rho)$ as the waveforms (base functions) in a successive approximation. As stated, the evolution is determined such that energy and mass are conserved, i.e.

$$
\left\{\begin{array}{l}
\tilde{\mathcal{H}}(\varphi, \gamma, \rho)=E_{0} \\
\tilde{\mathcal{C}}(\varphi, \gamma, \rho)=M_{0},
\end{array}\right.
$$

with $\tilde{\mathcal{H}}$ and $\tilde{\mathcal{C}}$ the energy and mass calculated for the solitary wave with tail. In the three dimensional parameter space $\varphi, \gamma, \rho$, the two conditions Eq. (33) have a clear geometrical meaning, stating that the evolution takes place in the intersection of the level set of the energy and that of the mass. This determines the variables $\gamma$ and $\rho$ as functions of position $\varphi$. Specifically, differentiating Eq. (33) with respect to $\varphi$ results in the set of (coupled) differential equations:

$$
\left\{\begin{array}{l}
\tilde{\mathcal{H}}_{\varphi}+\tilde{\mathcal{H}}_{\gamma} \cdot \gamma_{\varphi}+\tilde{\mathcal{H}}_{\rho} \cdot \rho_{\varphi}=0 \\
\tilde{\mathcal{C}}_{\varphi}+\tilde{\mathcal{C}}_{\gamma} \cdot \gamma_{\varphi}+\tilde{\mathcal{C}}_{\rho} \cdot \rho_{\varphi}=0
\end{array}\right.
$$

Dynamic equations for $\dot{\varphi}$ and $\dot{\rho}$ can be obtained by differentiating Eq. (33) with respect to $t$ and substituting Eq. (24) for $\dot{\gamma}$. 
Remark: The approximate evolution as described above differs somewhat from the analysis given by Newell [9]. The difference is in the update of the height of the tail. Newell finds $\gamma$ as a function of $\varphi$, say $\gamma_{\mathrm{N}}(\varphi)$, from energy conservation of the solitary-wave approximation (without modification of the tail), so $\mathcal{H}\left(\gamma_{N}(\varphi), \varphi\right)=$ $E_{0}$; then the value of $\rho$ is found from mass-conservation $\tilde{\mathcal{C}}\left(\gamma_{\mathrm{N}}(\varphi), \varphi, \rho\right)=m_{0}$. In contrast, here we derive the functions $\gamma$ and $\rho$ as functions of $\varphi$ from the two coupled equations (34), the intersecting level sets. It is clear that $\tilde{\mathcal{H}}_{\rho}=\int_{\sigma^{-1}(t)}^{\varphi} \mathcal{S}(\varphi, \gamma) \mathrm{d} x$ is of essential order. Hence, Newell's results differ from the above within the order of investigation.

We will not go into the details of the dynamic equations. We restrict to one observation. Suppose that after a while, the wave has passed the unevenness and that it continues to travel above a flat bottom, say $h(x)=h\left(\varphi_{1}\right)$ for $x \geq \varphi_{1}$. After passage, we have from Eq. (24) that $\gamma$, (the momentum of the solitary wave only) is constant and $\dot{\varphi}$ is just the velocity of the solitary wave above flat bottom $\dot{\varphi}=\lambda\left(\varphi_{1}\right)$. If $\Delta m$ is the total difference in mass between the solitary wave before and after passage, this mass should be incorporated in the tail, so, we have

$$
\Delta m=\int_{\sigma^{-1}(t)}^{\varphi(t)} \frac{\rho}{\sqrt{c(x)}} \mathrm{d} x=\tau \rho
$$

To see how the height of the tail then changes, we use mass conservation:

$$
\frac{\mathrm{d}}{\mathrm{d} t} \tilde{\mathcal{C}}=\tilde{\mathcal{C}}_{\varphi} \cdot \dot{\varphi}+\tilde{\mathcal{C}}_{\rho} \cdot \dot{\rho}=\left(\frac{22}{3 g}\left(\frac{\gamma}{4}\right)^{1 / 3} c^{5 / 6} c^{\prime}+\rho c^{-1 / 2}\right) \cdot \lambda(\varphi)+\tau \cdot \dot{\rho}=0 .
$$

Substituting Eq. (35) in Eq. (36) and putting $c^{\prime}=0$ gives

$$
\dot{\rho}=-\frac{1}{D} \rho^{2} \quad \text { with } \quad D=\frac{\sqrt{c(\varphi)}}{\lambda(\varphi)} \Delta m
$$

and the solution of Eq. (37) is

$$
\rho(t)=D \frac{1}{t}
$$

So, the height of the tail decreases inversially proportional with time, see Fig. 4 .

\subsection{Solitary waves above adjusting equilibrium surfaces}

A second modification to regain mass conservation is possible for bottom topographies that decay sufficiently fast at infinity. Specifically we will require the following integral to be convergent

$$
\int \frac{1}{c(x)} \mathrm{d} x \equiv \kappa<\infty
$$

In this case, the generalised equilibrium surface

$$
\eta_{\beta}(x)=\frac{\beta}{\sqrt{c(x)}}
$$

carries a finite amount of mass: $\mathcal{C}\left(\eta_{\beta}\right)=\beta \kappa$. This makes it possible to consider the superposition of a solitary wave and the generalised equilibrium surface:

$$
\breve{\mathcal{S}}(\varphi, \gamma, \beta)=\mathcal{S}(\varphi, \gamma)+\frac{\beta}{\sqrt{c(x)}} .
$$




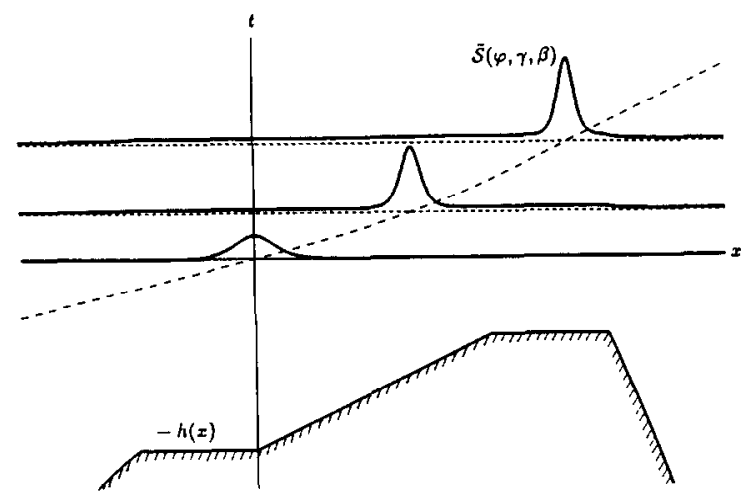

Fig. 5. Distorted solitary wave above generalised equilibrium surface.

Using $\gamma$ and $\beta$ as parameters to be adjusted, energy and mass conservation can both be satisfied, just like in the previous subsection, with $\beta$ replacing the parameter $\rho$. It should be noted that, although decreasing fast at infinity, the elevation described by $\eta_{\beta}$ does not have bounded support: in contrast to the tail, it extends to infinity before and after the solitary wave.

Since the change in mass carried by the solitary waves is of order $\mathcal{O}(\epsilon), \beta$ is of that order too. There is a mathematical justification for considering waves above such generalised equilibria. This becomes clear when investigating the physically motivated optimisation problem for solitary waves in which the mass conservation is taken as an additional constraint from the start on:

$$
\max _{\eta}\left\{\bar{H}(\varphi, \eta) \mid \bar{I}(\varphi, \eta)=\gamma, C(\eta)=m, \eta \in X_{\infty}\right\}
$$

with $\bar{H}(\varphi, \eta)$ and $\bar{I}(\varphi, \eta)$ given in Eqs. (15) and (16) respectively. (Note that $C(\eta)$ is not conserved for the $\mathrm{KdV}$ equation for flat bottom $h(\varphi)$; it changes in order $\mathcal{O}(\epsilon)$.) Solutions of Eq. (40) satisfy the equation

$$
\delta \bar{H}=\lambda \delta \bar{I}+\frac{\sigma}{\sqrt{c(x)}} \text { for some Lagrange multipliers } \lambda, \sigma \in R,
$$

explicitly

$$
\eta+\epsilon\left(\frac{1}{6} h(\varphi)^{2} \eta_{x x}+\frac{3}{4 h(\varphi)} \eta^{2}\right)=\frac{\lambda}{c(\varphi)} \eta+\frac{\sigma}{\sqrt{c(x)}}
$$

(From this equation, since $\eta \in X_{\infty}$, it is also clear that we need to assume that $c(x) \rightarrow \infty$ as $x \rightarrow \pm \infty$.) The existence of solutions of Eq. (41) will be dealt with in the appendix. Here, assuming the existence, let us look for an approximate solution of the form $\mathcal{S}(\varphi, \gamma, \beta)$ as suggested above.

Then $\mathcal{S}(\varphi, \gamma, \beta)$ will indeed be a solution of Eq. (41) if $\beta$ is taken according to

$$
\left(1-\frac{\lambda}{c(\varphi)}\right) \cdot \beta=\sigma+\mathcal{O}\left(\epsilon \beta^{2}\right)
$$

Note that from this it follows that $\sigma$ is of order $\mathcal{O}\left(\epsilon^{2}\right)$, and hence $\sigma / \sqrt{c(x)}$ is a perturbation in Eq. (41).

Using $\breve{S}(\varphi, \gamma, \beta)$ as base functions, energy and mass conservation define $\gamma$ and $\beta$ as functions of position $\varphi$ as before; dynamic equations can just as well be derived. We restrict ourselves to one observation. If in some interval we meet a flat bottom $\left(c^{\prime}=0\right)$, then from Eq. (24) it follows that $\gamma$ is constant and the velocity of the solitary wave is $\dot{\varphi}=\lambda(\varphi)$. Then, to see how the equilibrium changes, we use the mass conservation:

$$
\breve{\mathcal{C}}_{\varphi} \cdot \dot{\varphi}+\breve{C}_{\beta} \cdot \dot{\beta}=\mathbf{0},
$$


i.e.

$$
\frac{22}{3 g}\left(\frac{\gamma}{4}\right)^{1 / 3} c^{5 / 6} c^{\prime} \cdot \dot{\varphi}+\kappa \cdot \dot{\beta}=0
$$

Hence, when $c^{\prime}=0, \dot{\beta}=0$ : the height of the equilibrium is constant. This is to be expected, since above flat bottom, there is no change in mass that should be taken by the equilibrium.

\section{Distortion of cnoidal waves}

For the distortion of a periodic (cnoidal) wave due to topography, we use the method of Section 3, a quasihomogeneous approximation with uniform cnoidal waves. At a certain place $x=\varphi$, the distorted cnoidal wave is approximated by a uniform cnoidal wave for $\mathrm{KdV}$ equation for a flat bottom (14). Just as for solitary waves, given a certain wavelength, the cnoidal waves can be characterised as solutions of the constrained maximisation problem of maximising the energy on level sets of the momentum and mass. (Zero mass will be prescribed.) Explicit formulae for the profiles and velocity (expressed in elliptic functions) can be found in e.g. Ref. [3]. In this description, the wavelength should be prescribed a priori. For solutions of KdV-top the wavelength will not be constant. This can already be seen from the simplest linear equation (31). For solutions $\eta$ of Eq. (31), $\sqrt{c(x)} \eta$ are constant along characteristics $\mathrm{d} x / \mathrm{d} t=c(x)$ ( $\neq$ constant), which causes the changes in horizontal distances depending on the bottom topography. There is a simple way out to take account of this change in wavelength by scaling the horizontal coordinate $x$. To that end introduce a new variable

$$
\hat{x}=\int \frac{c_{0}}{c(x)} \mathrm{d} x
$$

and the corresponding elevation function $\hat{\eta}(\hat{x}, t)=\eta(x, t)$. In the new $\hat{x}, t$-variables, $\mathrm{d} \hat{x} / \mathrm{d} t=\left(c_{0} / c(x)\right)(\mathrm{d} x / \mathrm{d} t)$ $=c_{0}$, constant, which means that in this new variables the wavelength is constant.

Rewriting $H(\eta), I(\eta)$ and $C(\eta)$ in the new variables $\hat{x}$ and $\hat{\eta}$, and taking $g=1, c_{0}=1$ for simplicity, there results

$$
\begin{aligned}
& \hat{H}(\hat{\eta})=\int \frac{1}{2} c(\hat{x}) \hat{\eta}^{2}+\epsilon\left(-\frac{1}{12} c^{3}(\hat{x}) \hat{\eta}_{\hat{x}}^{2}+\frac{\hat{\eta}^{3}}{4 c(\hat{x})}\right) \mathrm{d} \hat{x}, \quad \hat{I}(\hat{\eta})=\int \frac{1}{2} \hat{\eta}^{2} \mathrm{~d} \hat{x}, \\
& \hat{C}(\hat{\eta})=\int \sqrt{c(\hat{x})} \hat{\eta} \mathrm{d} \hat{x},
\end{aligned}
$$

If $T$ denotes the period of $\hat{\eta}$, the uniform cnoidal wave above a flat bottom at height $h(x)=h(\varphi)$ is obtained from the maximisation problem

$$
\max _{\hat{\eta}}\{\overline{\hat{H}}(\varphi, \hat{\eta}) \mid \overline{\hat{I}}(\varphi, \hat{\eta})=\gamma, \overline{\hat{C}}(\varphi, \hat{\eta})=0, \hat{\eta} \text { is } T \text { periodic }\},
$$

with

$$
\begin{aligned}
& \overrightarrow{\hat{H}}(\varphi, \hat{\eta})=\int_{0}^{T} \frac{1}{2} c(\varphi) \hat{\eta}^{2}+\epsilon\left(-\frac{1}{12} c^{3}(\varphi) \eta_{\hat{x}}^{2}+\frac{\hat{\eta}^{3}}{4 c(\varphi)}\right) \mathrm{d} \hat{x}, \quad \overline{\hat{I}}(\varphi, \hat{\eta})=\int_{0}^{T} \frac{1}{2} \hat{\eta}^{2} \mathrm{~d} \hat{x}, \\
& \overline{\hat{C}}(\varphi, \hat{\eta})=\int_{0}^{T} \sqrt{c(\varphi)} \hat{\eta} \mathrm{d} \hat{x} .
\end{aligned}
$$




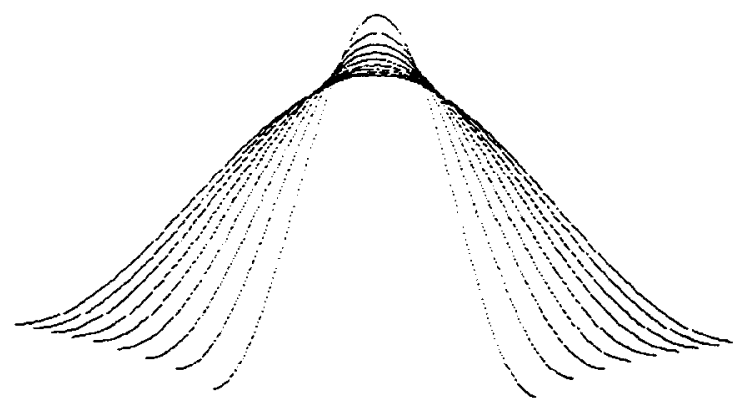

Fig. 6. Several uniform cnoidal waves centred at $x=0$ describing distortion of a cnoidal wave during run up.

Note that the restriction to waves with zero mass: $\bar{C}(\varphi, \eta)=0$, implies that the mass functional $C(\eta)$ is constant (equal to 0 ) for this approximation and there is no problem with mass conservation. The shape and the velocity are uniquely determined by the momentum value $\gamma$. For small values of $\gamma$ the solution is nearly sinusoidal, while for large values of $\gamma$ the solution is soliton-like with periodic peaks. According to the Lagrange multiplier rule, solutions of Eq. (44) satisfy

$$
\delta_{\eta} \overline{\hat{H}}=\lambda \delta_{\eta} \overline{\hat{I}}+\sigma \delta_{\eta} \overline{\hat{C}},
$$

for some multipliers $\lambda, \sigma \in R$. Eq. (46) is in fact the equation that is satisfied by cnoidal wave solution of Eq. (14) with velocity $\lambda$.

The problem (44) above can be replaced by the constrained minimisation problem of minimising the momentum at prescribed energy (and mass):

$$
\min _{\eta}\left\{\overline{\hat{I}}(\varphi, \hat{\eta}) \mid \overline{\hat{H}}(\varphi, \hat{\eta})=E_{0}, \overline{\hat{C}}(\varphi, \hat{\eta})=0, \hat{\eta} \text { is } T \text { periodic }\right\} \text {. }
$$

This formulation is easier to use in numerical calculations, where Eq. (47) is solved numerically to prevent to use the rather awkward analytical expressions (44). Then using a simple piecewise linear topography, a linear transition between two constant depths as in Fig. 4, we show the distortion of cnoidal wave during run up. The calculated profiles for several values of $\varphi$ are shown in Fig. 6. It shows a behaviour that can be expected: amplitude increase and wavelength and velocity decrease during run up.

\section{Appendix}

In this appendix, we prove directly the existence of solution of Eq. (40), i.e. the existence of solitary wave solution above the equilibrium $\beta / \sqrt{c(x)}$. First, rewrite Eq. (41) as a perturbed potential equation

$$
\begin{aligned}
& \epsilon \eta_{x x}=-V^{\prime}(\eta)+C \frac{\sigma}{\sqrt{c(x)}}, \\
& \quad \text { with } \quad V(\eta)=-\frac{A}{2} \eta^{2}+\frac{B}{3} \eta^{3} \quad \text { and } A=\frac{6}{h^{2}(\varphi)}\left(\frac{\lambda}{c(\varphi)}-1\right), \quad B=\frac{9}{2 h^{3}(\varphi)} \epsilon, \quad C=\frac{6}{h^{2}(\varphi)} .
\end{aligned}
$$

The term $C \sigma / \sqrt{c(x)}$ is of order $\mathcal{O}\left(\epsilon^{2}\right)$, so it is a perturbation term. The graph of $V(\eta)$ is given in Fig. 7. It is clear that there is a homoclinic orbit which is in fact the solitary wave solution $\mathcal{S}(\varphi, \gamma)$ of the unperturbed system $(\sigma=0)$. We will show that under a certain condition of $c(x)$, there exists a perturbed homoclinic 

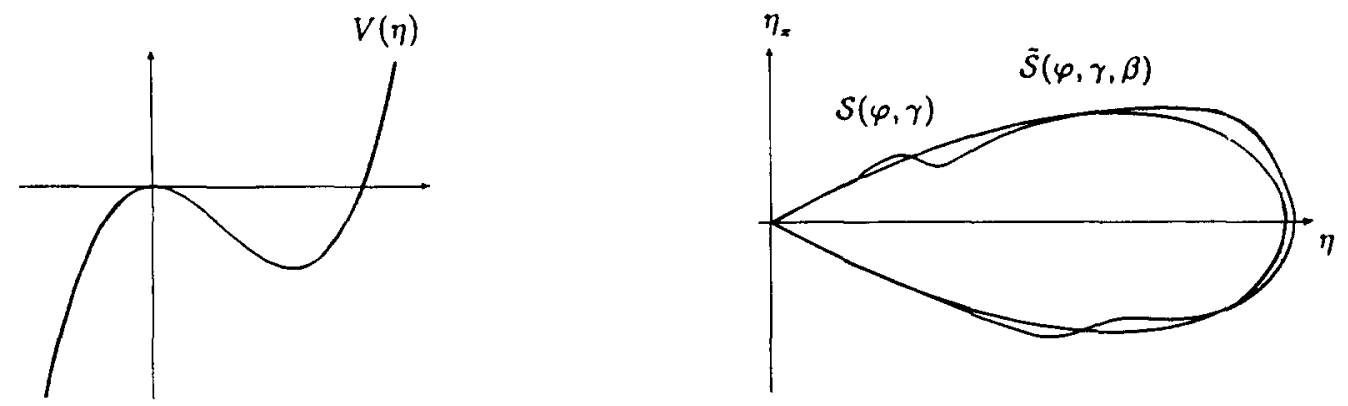

Fig. 7. (Left) Potential function $V(\eta)$.

Fig. 8. (Right) Homoclinic orbit and the perturbed homoclinic orbit.

orbit (i.e. a solitary wave above the equilibrium surface) of the perturbed system (A.1), see Fig. 8. If $c(x)$ were periodic, we would use the standard Melnikov method, see Ref. [6]. Since here $c(x)$ is not periodic but $c(x) \rightarrow 0$ as $x \rightarrow \pm \infty$, we give a complete proof of the following, Melnikov-type lemma.

Lemma 1. Perturbed homoclinic solutions of Eq. (A.1) exist if $c(x)$ satisfies

$$
\int \frac{1}{\sqrt{c(x)}} \frac{\mathrm{d}}{\mathrm{d} x} \mathcal{S}(\varphi, \gamma)(x-\psi) \mathrm{d} x=0, \quad \text { for some } \psi \in R
$$

Before proving the lemma, we formulate the main consequence.

Proposition 1. If $c(x)$ increases monotonically to infinity as $|x| \rightarrow \infty$, condition (A.3) is satisfied, and hence there exists a solution of Eq. (A.1).

Proof of the proposition: Define the function

$$
P(\psi) \equiv \int \frac{1}{\sqrt{c(x)}} \frac{\mathrm{d}}{\mathrm{d} x} \mathcal{S}(\varphi, \gamma)(x-\psi) \mathrm{d} x=\int \frac{1}{\sqrt{c(x+\psi)}} \frac{\mathrm{d}}{\mathrm{d} x} \mathcal{S}(\varphi, \gamma)(x) \mathrm{d} x .
$$

This continuous function $P(\psi)$ will have a zero if it has different sign at plus and minus infinity. Observe that $\mathrm{d} / \mathrm{d} x \mathcal{S}(\varphi, \gamma)(x)$ is an odd function around $x=0$, it is positive for $x<0$ and negative for $x>0$, and it is exponentially small outside an interval around $x=0$. Given the monotonicity of $c$ for large value of $|x|$ (a sufficient, but not necessary condition), it can be concluded that $P(\psi)>0$ for $\psi \rightarrow \infty$ and $P(\psi)<0$ for $\psi \rightarrow-\infty$ which proves the statement.

Proof of the lemma: Consider

$$
F(\eta, \sigma):=-\epsilon \eta_{x x}-V^{\prime}(\eta)+C \frac{\sigma}{\sqrt{c(x)}} .
$$

It is clear that $F(\mathcal{S}(\varphi, \gamma), 0)=0$. Expanding $F(\eta, \sigma)$ around $(\mathcal{S}(\varphi, \gamma), 0)$ gives

$$
\begin{aligned}
& F(\eta, \sigma)=D_{\eta} F(\mathcal{S}(\varphi, \gamma), 0)(\eta-\mathcal{S})+D_{\sigma} F(\mathcal{S}(\varphi, \gamma), 0) \sigma+\text { h.o.t. }=L(\mathcal{S})(\eta-\mathcal{S})+\frac{C}{\sqrt{c(x)}} \sigma+\text { h.o.t. } \\
& \quad \text { with } L(\mathcal{S})=-\epsilon \partial_{x}^{2}-V^{\prime \prime}(\mathcal{S}) .
\end{aligned}
$$

Neglecting higher order terms and investigating the existence of $\eta$ such that $F(\eta, \sigma)=0$ is in fact investigating existence of solution (41). Equivalently, this is proving the existence of $\eta$ such that 


$$
L(\mathcal{S})(\eta-\mathcal{S})=-\sigma \frac{C}{\sqrt{c(x)}} .
$$

Eq. (A.5) is a scattering problem. Using Fredholm alternative, the existence of solution of Eq. (A.5) is guaranteed if and only if

$$
-\frac{C}{\sqrt{c(x)}} \in \operatorname{Range}(L(\mathcal{S})) \quad \text { or } \quad-\frac{C}{\sqrt{c(x)}} \perp \operatorname{Kernel}\left(L(\mathcal{S})^{*}\right) \quad \text { or } \quad-\frac{C}{\sqrt{c(x)}} \perp \operatorname{Kernel}(L(\mathcal{S})),(\text { A.6) }
$$

since Range $(L(\mathcal{S}))$ is closed and $L(\mathcal{S})$ is symmetric $\left(L(\mathcal{S})^{*}=L(\mathcal{S})\right)$. A solitary wave $\mathcal{S}(\varphi, \gamma)(x)$ satisfies

$$
\epsilon \eta_{x x}+V^{\prime}(\eta)=0
$$

and so does its translation $\mathcal{S}(\varphi, \gamma)(x-\psi)$, for all $\psi \in R$ (translation invariant). Differentiating (A.7) with respect to $x$ shows that $\mathrm{d} / \mathrm{d} x \mathcal{S}(\varphi, \gamma)(x-\psi)$ is in the kernel of $L(\mathcal{S}(\varphi, \gamma))(x-\psi))$. The third condition in Eq. (A.6) is then precisely the condition (A.3) in the lemma. It remains to show that Eq. (A.3) is the only condition. To that end, use the fact that the scattering problem (A.5) has discrete spectrum with a finite number of simple eigenvalues. Hence, $\mathcal{S}_{x}(\varphi, \gamma)(x-\psi)$ being the eigenfunction with the eigenvalue $\sigma=0$, it is the only eigenfunction and spans the kernel of $L(\mathcal{S})$. Hence, Eq. (A.3) is the only condition for $c(x)$. q.e.d.

\section{References}

[1] L.J.F. Broer, "On the Hamiltonian theory of surface waves", Appl. Sci. Res. 29, 430-446 (1974).

[2] L.J.F. Broer, E. van Groesen, J. Timmers, "Stable model equations for long water waves", Appl. Sci. Res. 32, 619-634 (1976).

[3] P.G. Drazin, R.S. Johnson, Solitons: an introduction, Cambridge University Press, Cambridge, New York, Melbourne, Sydney, (1989).

[4] E. van Groesen, E.M. de Jager, "Mathematical structures in continuous dynamical systems", in series: Studies in Mathematical Physics, vol. 6, North-Holland, Elsevier, Amsterdam (1994).

[5] E. van Groesen, S.R. Pudjaprasetya, "Uni-directional Waves over slowly varying bottom. Part I: Derivation of a KdV-type of equation", Wave Motion 18, 345-370 (1993).

[6] J. Guckenheimer, P. Holmes, "Nonlinear oscillations, dynamical systems and bifurcations of vector fields", Springer-Verlag, New York, (1983).

[7] V.I. Karpman, E.M. Maslov, "Structure of tails produced under the action of perturbation on solitons", Sov. Phys. JETP 48, 252-259 (1978).

[8] C.J. Knickerbocker, A.C. Newell, "Shelves and the Korteweg-de Vries equation", J. Fluid Mech. 98, 803-818 (1980).

[9] A.C. Newell, Soliton in mathematics and physics, Society for Industrial and Applied Mathematics, Philadelphia, Pennsylvania, (1985).

[10] V.E. Zakharov, "Stability of periodic waves of finite amplitude on the surface of a deep fluid", J. Appl. Mech. Tech. Phys. 2, 190-194, (1968). 